\title{
Effect of implementation of guidelines on assessment and diagnosis of vertebral fractures in patients older than 50 years with a recent non-vertebral fracture
}

\author{
R. Y. van der Velde ${ }^{1,2}$ - S. P. G. Bours ${ }^{3}$ - C. E. Wyers ${ }^{1,2}$ - W. F. Lems ${ }^{4}$. \\ P. P. M. M. Geusens ${ }^{3,5}$ - J. P. W. van den Bergh ${ }^{1,2,5}$
}

Received: 2 February 2017 / Accepted: 4 July 2017 /Published online: 26 July 2017

(C) The Author(s) 2017. This article is an open access publication

\begin{abstract}
Summary We evaluated the impact of a new Dutch guideline on systematic implementation of densitometric Vertebral Fracture Assessment (VFA) in patients with a recent nonvertebral fracture. Systematic implementation resulted in a significant increase of VFA, diagnosis of vertebral fractures (VFs), and percentage of patients eligible for treatment.

Introduction VFs are underdiagnosed although they are important predictors of fracture risk, independent of age and bone mineral density (BMD). The Dutch guideline on osteoporosis and fracture prevention recommends VFA in all patients aged $>50$ years with a recent non-VF. Our aim was to evaluate the effect of systematic implementation of densitometric VFA in patients with a recent non-VF at the fracture liaison service (FLS).

Methods VFA was performed on lateral images of the spine using dual-energy X-ray absorptiometry (DXA) and graded according to Genant using Spine Analyzer software.
\end{abstract}

Robert Y. van der Velde and Sandrine P.G. Bours contributed equally to this manuscript.

R. Y. van der Velde

robertvandervelde@planet.nl

1 Department of Internal Medicine, VieCuri Medical Centre for North Limburg, Tegelseweg 210, 5912 BL Venlo, The Netherlands

2 Department of Internal Medicine, NUTRIM School of Nutrition and Translational Research in Metabolism, Maastricht UMC, Maastricht, The Netherlands

3 Department of Rheumatology, CAPHRI, Maastricht UMC, Maastricht, The Netherlands

4 Department of Rheumatology, VU Medical Centre, Amsterdam, The Netherlands

5 Biomedical Research Centre, University of Hasselt, Hasselt, Belgium
Results We evaluated 582 patients before and 484 after implementation (mean age 67 and 66 years; 71 and $74 \%$ women, respectively). Performing VFA increased from 4.6 to $97.1 \%$ $(p<0.001)$ and the diagnosis of VFs from 2.2 to $26.2 \%$ for grade $\geq 1(p<0.001)$ and from 0.9 to $14.7 \%$ for grade $\geq 2$ $(p<0.001)$. Prevalence of VFs increased with age $(5.2 \%$ in $50-59$-year olds to $27.8 \%$ in $80+$-year olds, $p<0.001$ ), but was similar for both genders, non-VF locations, and BMD. Including patients with osteopenia and a VF increased the percentage of patients eligible for treatment by a quarter, from $31.0 \%$ in the pre-guideline to $38.4 \%$ in the post-guideline cohort.

Conclusions Systematic guideline implementation resulted in a significant increase of VFA, diagnosis of VFs, and percentage of patients eligible for treatment. VFA contributes to documenting the high prevalence of VFs in patients visiting the FLS with a non-VF in both genders, at any age, non-VF location, and BMD.

Keywords BMD · Fracture liaison service - Guideline · Osteoporosis $\cdot$ Vertebral fracture $\cdot$ VFA

\section{Introduction}

Vertebral fractures (VFs) are the most frequently occurring osteoporotic fractures [1-3]. They are underdiagnosed [4, 5] because only one third of patients with VFs present with an acute symptomatic episode [6]. Even in the case of acute back pain or height loss, imaging of the spine is not always performed; additionally, when radiographs are available, VFs are also often overlooked $[4,7]$. The presence, number, and severity of VFs are strong predictors of future fracture risk, independent of age and bone mineral density (BMD) $[8,9]$. VFs are frequently present in patients with a recent non-VF 
( $>13$ to $>20 \%$, depending on the grading of deformity) [10, $11]$ and in patients with osteopenia with or without a recent fracture [12]. Therefore, the Dutch guideline on osteoporosis and fracture prevention released in 2011 (www.cbo.nl) recommended systematic evaluation of VFs in patients with a recent non-VF and a BMD T-score of $<-1.0$ and $>-2.5$. In those osteopenic patients who have a grade 2 or $3 \mathrm{VF}$, the guideline recommends the initiation of anti-osteoporotic medication.

The aim of our study was to compare the proportion of patients with a recent non-VF at the fracture liaison service (FLS) that had Vertebral Fracture Assessment (VFA) and the prevalence of VFs, before and after implementation of the guideline, as well as the impact the diagnosis of VF had on the percentage of patients that was diagnosed to have osteoporosis and to be eligible for treatment.

\section{Materials and methods}

\section{Study population}

Patients included in our study were consecutive men and women aged 50 years and older with a recent low-energy non-VF visiting the FLS of VieCuri Medical Center for Northern Limburg (VieCuri) and Maastricht University Medical Center (MUMC). Before guideline implementation, VFA in the FLS at VieCuri was performed only on indication of the clinician; while in MUMC, there was temporarily no FLS because of financial restrictions, but VFA was available and performed on indication of the surgeon who treated the fracture. After guideline implementation, an FLS with densitometric VFA was available in both centers. The study protocol was approved by the local ethical committee of VieCuri (number CEM/11091).

\section{Methods}

Both hospitals had the same equipment available for BMD measurement at the lumbar spine and hip and lateral spine imaging, using dual-energy X-ray absorptiometry (DXA) (Hologic QDR 4500, Hologic, Bedford, MA, USA). BMD measurements were classified according to the lowest value of T-score in the total hip/femoral neck or lumbar spine: osteoporosis as $\mathrm{T}$-score $\leq-2.5$, osteopenia as T-score between -2.5 and -1.0 , and normal BMD as T-score $\geq-1.0$.

After collecting all pre- and post-guideline densitometric lateral spine VFA images, central evaluation by the same experienced researcher was performed using a morphometry software program (Spine Analyzer, Optasia Medical, Manchester, UK) [13]. First, the image quality was evaluated and the vertebrae were labeled, starting with the identification of the fourth lumbar vertebra. Subsequently, the evaluable vertebrae were determined. A vertebra was considered evaluable if the posterior and anterior cortices and both endplates were fully and clearly visible. If this was not the case, the vertebra was not evaluated. Images were visually inspected for the presence of a VF. The researcher studied both the vertebral shape and the appearance of the end plate in order to differentiate between VFs and vertebrae with other deformities, e.g., degenerative changes or Scheuermann's disease, only true VFs were counted. When a vertebra was visually suspect for a VF, the point placement of the Spine Analyzer software program was used to measure the anterior, mid-vertebral, and posterior height of the vertebra that had been selected on visual inspection. The point placement was edited occasionally when this was deemed necessary, but we did not keep a record on how often this was done. The vertebral fractures were then graded according to the grading of Genant et al. [14] as grade $0,<20 \%$ reduction in expected vertebral body height at the anterior, mid, or posterior location; grade $1,20-24 \%$; grade $2,25-39 \%$; or grade $3, \geq 40 \%$ reduction, respectively.

Non-VFs were classified according to the method of Center [15] into hip fractures, major fractures (multiple rib, humerus, pelvis, distal femur, and proximal tibia), and minor fractures (all remaining fractures except fingers and toes).

\section{Statistical analysis}

Data were analyzed using Independent samples T-test, chisquare statistics, and odds ratios. Subgroup analyses were performed for BMD (normal versus osteopenia versus osteoporosis) and for fracture type according to Center (minor versus major versus hip fracture). Statistical analyses were performed using SPSS for Mac (version 21.0, IBM SPSS Statistics, USA).

\section{Results}

In total, 1066 consecutive patients (768 women, $298 \mathrm{men}$ ) were evaluated, 582 (411 women, 171 men) before and 484 (357 women, 127 men) after guideline implementation (Fig. 1). Patients after implementation were somewhat younger and sustained less often a major or hip fracture, but BMI was similar (Table 1). BMD measurements before implementation were performed in all patients in VieCuri and in $30(11 \%)$ in MUMC (in total 338 out of $582(58.1 \%)$ patients) and in all patients $(p<0.001)$ after implementation. Before and after implementation, the prevalence of osteoporosis, osteopenia, and normal BMD was similar (Table 1). VFA was performed in $4.6 \%$ before implementation and in $97.1 \%$ of patients after implementation $(p<0.001)$ (Fig. 1). Within the vertebral range $\mathrm{Th} 4$ to $\mathrm{L} 4$, the percentage of evaluable vertebrae 
Fig. 1 Flowchart with the number and percentage of patients per center with a dualenergy X-ray absorptiometry (DXA), a Vertebral Fracture Assessment (VFA), and at least one newly diagnosed grade 2 or 3 vertebral fracture (VF) before and after the introduction of systematic VFA.

VieCuri $=$ VieCuri Medical

Center; MUMC = Maastricht University Medical Center

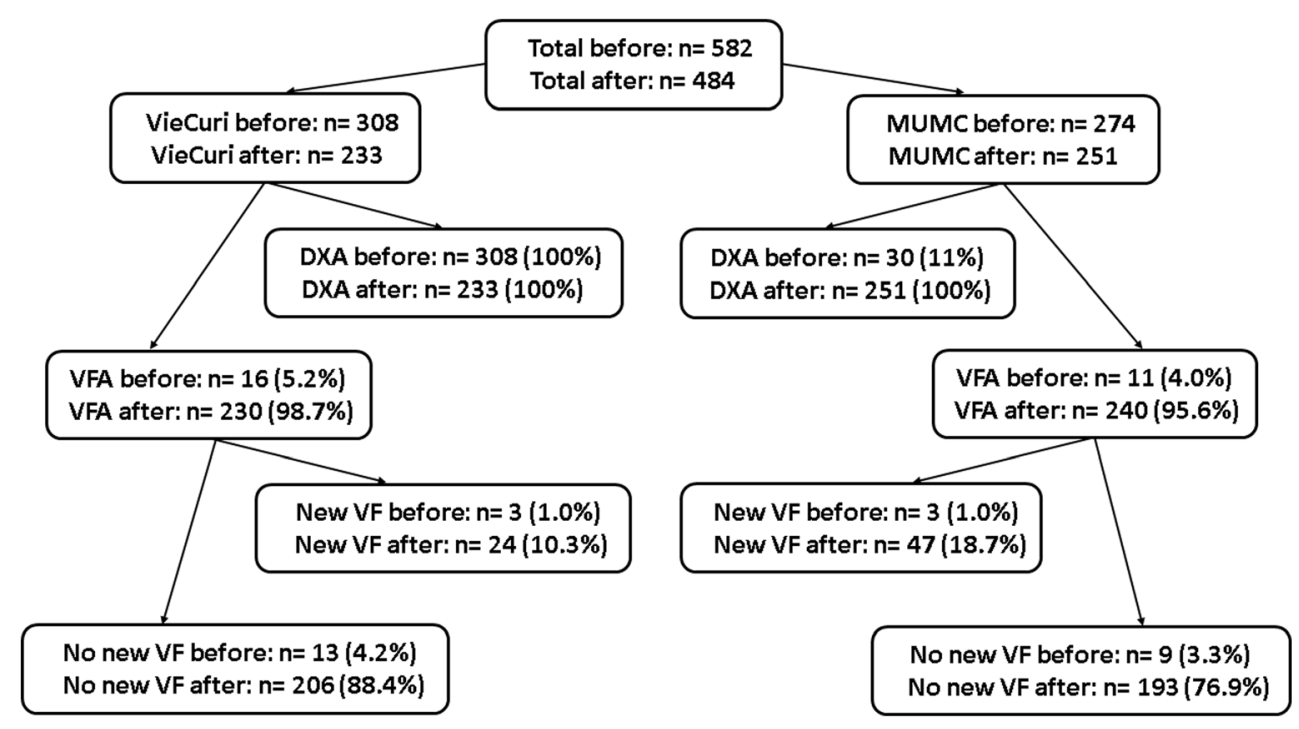

Table 1 Baseline characteristics of the study population before and after the implementation of Vertebral Fracture Assessment according to the Dutch guideline

\begin{tabular}{|c|c|c|c|}
\hline & $\begin{array}{l}\text { Before VFA } \\
\text { implementation } \\
(n=582)\end{array}$ & $\begin{array}{l}\text { After VFA } \\
\text { implementation } \\
(n=484)\end{array}$ & $p$ value \\
\hline \multicolumn{4}{|l|}{ Gender and age } \\
\hline Women, $n(\%)$ & $411(70.6)$ & $357(73.8)$ & \\
\hline Men, $n(\%)$ & $171(29.4)$ & $127(26.2)$ & \\
\hline Age, mean (SD) & $67.0(10.6)$ & $65.9(9.0)$ & $p<0.001$ \\
\hline Fracture location & & & $p=0.002$ \\
\hline Minor $n(\%)$ & $386(66.3)$ & $344(71.1)$ & \\
\hline Major $n(\%)$ & $126(21.6)$ & $107(22.1)$ & \\
\hline Hip $n(\%)$ & $70(12.0)$ & $33(6.8)$ & \\
\hline $\mathrm{BMD}^{\mathrm{a}}$ & & & $p=0.065$ \\
\hline Normal $n(\%)$ & $71(21.0)$ & $91(18.8)$ & \\
\hline Osteopenia $n(\%)$ & $163(48.2)$ & 235 (48.6) & \\
\hline Osteoporosis $n(\%)$ & $104(30.8)$ & $158(32.6)$ & \\
\hline \multicolumn{4}{|l|}{ Weight and height } \\
\hline $\begin{array}{l}\text { Weight }^{\mathrm{b}}(\mathrm{kg}), \text { mean } \\
\text { (SD) }\end{array}$ & $74.5(13.8)$ & 72.5 (13.9) & $p=0.651$ \\
\hline $\begin{array}{l}\mathrm{Height}^{\mathrm{c}}(\mathrm{m}), \text { mean } \\
\quad(\mathrm{SD})\end{array}$ & $1.68(0.9)$ & $1.67(1.2)$ & $p=0.146$ \\
\hline $\begin{array}{l}\text { BMI }(\mathrm{kg} / \mathrm{m}) \text {, mean } \\
\quad(\mathrm{SD})\end{array}$ & $26.4(4.4)$ & $26.0(3.9)$ & $p=0.363$ \\
\hline
\end{tabular}

VFA Vertebral Fracture Assessment

${ }^{a}$ DXA measurement to assess BMD was performed in 338 (58.1\%) patients before guideline and in 484 (100\%) after

${ }^{\mathrm{b}}$ Weight was known in 256 patients before guideline and in 234 patients after guideline

${ }^{\mathrm{c}}$ Height was known in 257 patients before guideline and in 245 patients after guideline increased from $20 \%$ at Th4 to $>70 \%$ at Th8 and Th9 and to $>90 \%$ from TH10 to L4 (Fig. 2).

The diagnosis of a VF increased from 2.2 to $26.2 \%$ for grade $\mathrm{VF} \geq 1 \quad(p<0.001)$ and from 0.9 to $14.7 \%$ for grade $\geq 2$ VF $(p<0.001$ ) (Table 2). The prevalence of VFs grade $\geq 2$ increased with age (Table 2) but was similar for both sexes, non-VF locations, and BMD (Table 2). After implementation, in patients with osteopenia, $13.6 \%$ had at least one grade $\geq 2$ VF (Table 2).

The odds ratio for the presence of a grade $\geq 2 \mathrm{VF}$ was nearly twofold higher $(p=0.043)$ in patients with hip or major fracture compared to that in patients with a minor fracture (Table 2).

\section{Discussion}

After systematic implementation of VFA in FLS patients with a recent non-VF according to the Dutch guideline for osteoporosis, performing VFA increased nearly 20 -fold, diagnosis of a VF of grade $\geq 2$ increased 15-fold, and one out of six patients was diagnosed with a grade $\geq 2 \mathrm{VF}$. As a result, the total percentage of patients diagnosed to have osteoporosis and therefore eligible for treatment according to the Dutch guideline increased by one quarter.

The VF prevalence in our study is in accordance with that of two other studies in patients with a non-VF: $20-25 \%$ for grade $\geq 1$ and $13-17 \%$ for grade $\geq 2[10,11]$. The prevalence of VFs in our FLS cohort increased with age; the prevalence of grade $\geq 2$ VF increased more than fivefold between the ages of 50 and $80+$. A similar increase was found by others [10, 11, 16]. Remarkably, the prevalence of VF was similar between genders, non-VF locations, and BMD levels. The similar 
Fig. 2 Percentage of evaluable vertebrae on Vertebral Fracture Assessment (VFA) and of grade 1 or grades 2 and 3 vertebral fractures

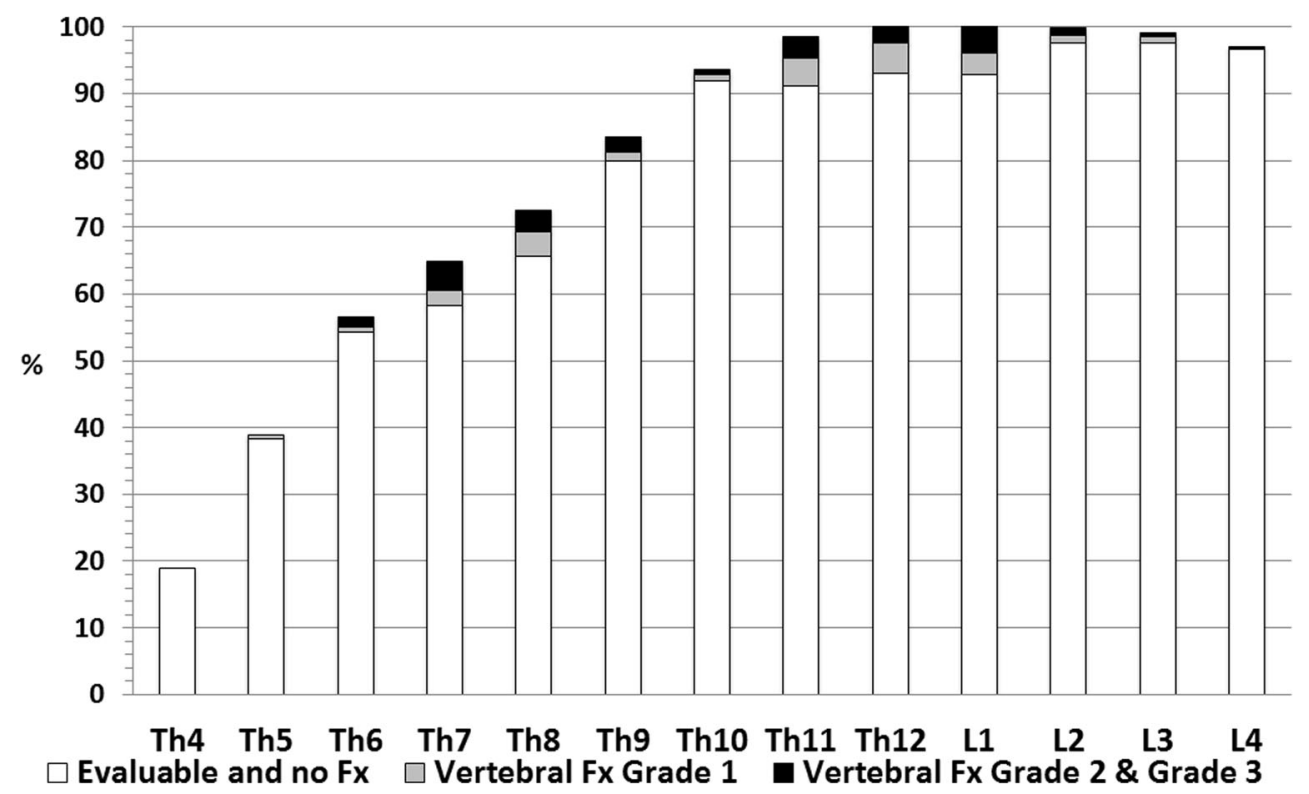

prevalence of VF seen in women and men in our study contrasts the higher VF prevalence found in women in many studies [17], but other authors also found a similar prevalence in both genders $[11,18,19]$ or even a higher prevalence in men [20], which was also seen in a recent Dutch study [21]. In contrast to others $[10,11]$, we found no significant difference in the prevalence of VFs grade $\geq 1$ between different non-VF locations but direct comparison to the other studies is difficult since we studied three NVF groups instead of individual NVF sites. Our results underscore the importance of performing VFA even after minor presenting fractures. We found VFs grade $\geq 2$ in any BMD category, with a non-significant trend of higher prevalence in patients with osteoporosis (18\%) compared to patients with a normal BMD (12\%). These results correspond well with those found by other authors $[12,21]$. In contrast, Gallagher et al. [10] reported VFs grade $\geq 2$ in $9 \%$ of patients with normal BMD, in $13 \%$ with osteopenia, and in $34 \%$ with osteoporosis. However, in that study, only lumbar spine BMD was reported, while we used the lowest T-score at the spine, hip, or femoral neck. Howat et al. [11] reported that grades 2 and 3 VFs correlated with spine and hip BMD in women, and in men only grade 3 VFs when compared to patients with a normal BMD. In spite of these differences in prevalence of VFs between studies, the results indicate that
Table 2 Percentage of postguideline patients with $\geq 1$ grades $1,2,3,1$ or 2 or 3 , and 2 or $3 \mathrm{VFs}$

\begin{tabular}{llllll}
\hline & Grade 1 & Grade 2 & Grade 3 & Grade 2 or 3 & Grade 1, 2, or 3 \\
\hline Gender & $p=0.56$ & $p=0.88$ & $p=0.91$ & $p=0.74$ & $p=0.19$ \\
Women & $14.4 \%$ & $11.4 \%$ & $3.3 \%$ & $13.2 \%$ & $24.1 \%$ \\
Men & $20.8 \%$ & $13.9 \%$ & $2.8 \%$ & $16.7 \%$ & $32.3 \%$ \\
Age & $p=0.12$ & $p=0.00$ & $p=0.00$ & $p=0.00$ & $p=0.00$ \\
50-59 years & $11.1 \%$ & $4.4 \%$ & $0.7 \%$ & $5.2 \%$ & $14.8 \%$ \\
$60-69$ years & $14.1 \%$ & $16.8 \%$ & $1.6 \%$ & $16.8 \%$ & $26.5 \%$ \\
$70-79$ years & $20.3 \%$ & $12.5 \%$ & $6.3 \%$ & $18.0 \%$ & 34.45 \\
$80+$ years & $19.4 \%$ & $22.2 \%$ & $11.1 \%$ & $27.8 \%$ & $38.9 \%$ \\
Fracture location & $p=0.44$ & $p=0.15$ & $p=0.15$ & $p=0.07$ & $p=0.29$ \\
Minor & $14.5 \%$ & $10.8 \%$ & $2.3 \%$ & $12.2 \%$ & $24.4 \%$ \\
Major & $16.8 \%$ & $17.8 \%$ & $5.6 \%$ & $21.5 \%$ & $30.8 \%$ \\
Hip & $18.2 \%$ & $15.2 \%$ & $6.1 \%$ & $18.2 \%$ & $30.3 \%$ \\
BMD & $p=0.42$ & $p=0.63$ & $p=0.54$ & $p=0.39$ & $p=0.44$ \\
Normal & $11.0 \%$ & $11.0 \%$ & $2.2 \%$ & $12.1 \%$ & $20.9 \%$ \\
Osteopenia & $15.7 \%$ & $12.3 \%$ & $3.0 \%$ & $13.6 \%$ & $27.7 \%$ \\
Osteoporosis & $17.1 \%$ & $13.9 \%$ & $4.4 \%$ & $17.7 \%$ & $27.2 \%$ \\
\hline
\end{tabular}


VFs are frequently present at any BMD in patients with a recent non-VF.

In the group of patients with a BMD score in the osteopenic range, $13.6 \%$ had at least one grade $\geq 2 \mathrm{VF}$, constituting a diagnosis of osteoporosis and an indication for treatment according to the Dutch guideline (www.cbo.nl). If treatment decisions would have been based on BMD results only, 31. $0 \%$ of the post-guideline patients would be eligible for treatment because of osteoporosis. With implementation of VFA, this increases by a quarter to $38.4 \%$. Our finding underscores the limitation of using a screening strategy based on BMD measured by DXA, as was also found by other authors [22, 23]. We also found VFs in patients with a normal BMD but the Dutch guideline does not recommend performing VFA routinely in these patients; so, in daily practice, these patients would not have been treated (www.cbo.nl).

Our results can be compared with the results of two other Dutch studies. Netelenbos et al. [24] found a $43 \%$ increase in the number of patients who qualified for osteoporosis when based on BMD + spine radiograph instead of based on BMD alone, considerably higher than in our study which can probably be explained by the fact that in the study by Netelenbos et al. VFs of all grades were counted as an indication for treatment (instead of only grade $\geq 2$ as stated in the Dutch guideline). In our study, of patients with osteopenia, $27.7 \%$ had at least one grade 1,2 , or grade $3 \mathrm{VF}$, so the number of patients from our post-group who would qualify for osteoporosis treatment would be $41 \%$ higher than when based on BMD alone, corresponding well with the results from Netelenbos et al. In another study from the Netherlands [21], in the subgroup with osteopenia, $21 \%$ had a vertebral fracture, $55 \%$ of those were grade 2 or 3 , which increased the number of patients eligible for treatment by one fifth from 27 to $32 \%$ when the VFA results were considered in the treatment decision, in keeping with our results. In a study from Hull (UK) in women over the age of $65,20 \%$ of osteopenic women had at least one grade 2 or grade 3 vertebral fracture [25]. In addition to the $17 \%$ of women with osteoporosis, the application of VFA identified another $11 \%$ of women with an indication for treatment, a greater increase probably explained by the higher age of the patients [25]. Results similar to those of our study were seen in a study from Glasgow (UK) in which overall in $25 \%$ of patients, a previously undiagnosed grade 1 , 2 , or 3 vertebral deformity was found [10]. In osteopenic patients, this was $20 \%$, of which in $13 \%$ there was a grade 2 or 3 vertebral fracture. In this study, $28 \%$ had a treatment indication based on BMD (osteoporosis) and $9 \%$ of patients were likely to have had a change in management based on their vertebral deformity status. In a study conducted in the USA [12], VFs were found in $18 \%$ of asymptomatic postmenopausal women. In this study, between 26 and $60 \%$ of osteoporotic patients could have potentially been missed if the diagnosis had been based on BMD alone. By contrast, in a study from Glasgow (UK), the authors found that VFA does identify a substantial burden of prevalent vertebral fractures that were not known before (about 20\%), but this (in 3\%) seldom influenced the need for treatment after a nonvertebral fracture [11]. This fact can be explained by the guideline used in this center, according to which in patients over the age of 65 , the BMD threshold is $\leq-2$ thereby limiting the number of osteopenic patients that would only get medical treatment based on diagnosis of VFs through VFA. In a recent study from Sheffield [26], in $11 \%$ of patients undergoing VFA, one or more vertebral fractures were found but this finding changed the management in only $3 \%$. This lower percentage can be explained by two factors: firstly, in this study, the focus was only on previously unidentified VFs diagnosed through VFA scans; and secondly, the methodology used in this study to identify VF (the ABQ method) is known to identify fewer VFs compared with other techniques [27].

The strength of our study is that the same device for DXA/ VFA was used in both centers, and that all images were examined by the same experienced investigator using the precise and accurate Spine Analyzer software (www.cbo.nl). Limitations are slight differences in patient characteristics before and after implementation of the guideline, reflecting the real-world response rate of patients invited to the FLS. Only limited vertebrae could be evaluated above the Th7 level. Possibly with today's DXA technology (with improved hardware and software), a higher proportion of vertebrae above the $\mathrm{Th} 7$ level could have been evaluated. However, since most osteoporotic VFs occur at the lower spine region, this will not have influenced the results much. Another limitation is the possibility of overdiagnosing VFs, but the VFA evaluation was performed by an experienced researcher, carefully excluding non-VF deformities.

In conclusion, we have found that systematic implementation of the guideline resulted in a significant increase in the diagnosis of VFs. VFA contributes to documenting the high prevalence of VFs in FLS patients with a non-VF in both genders, at any age, non-VF location, and BMD level. The finding of a grade 2 or $3 \mathrm{VF}$ in patients with osteopenia has direct clinical implications, increasing the number of patients in the total cohort eligible for treatment by one quarter.

Acknowledgments Funding: the research for this article was partly funded by the Eli Lilly Company, under grant number: VFA, B3D-UT0029. The funding source had no role in this manuscript.

\section{Compliance with ethical standards}

Conflicts of interest P. Geusens has received grants from Amgen, Pfizer, MSD, UCB, Abbott, BMS, Novartis, Roche, and Will Pharma as well as a grant and a speaker honorarium from Eli Lilly. The other authors declare they have no conflict of interest. 
Statement of human rights and ethical approval The study protocol was approved by the local ethical committee of VieCuri (number CEM/ 11091) and the study has been performed in accordance with the ethical standards as laid down in the 1964 Declaration of Helsinki and its later amendments or comparable ethical standards. Informed consent was obtained from all individuals participants included in the study.

Open Access This article is distributed under the terms of the Creative Commons Attribution-NonCommercial 4.0 International License (http:// creativecommons.org/licenses/by-nc/4.0/), which permits any noncommercial use, distribution, and reproduction in any medium, provided you give appropriate credit to the original author(s) and the source, provide a link to the Creative Commons license, and indicate if changes were made.

\section{References}

1. Cauley JA, Palermo L, Vogt M et al (2008) Prevalent vertebral fractures in black women and white women. J Bone Miner Res 23(9): 1458-1467

2. Melton LJ III, Lane AW, Cooper C, Eastell R, O'Fallon WM, Riggs BL (1993) Prevalence and incidence of vertebral deformities. Osteoporos Int 3(3):113-119

3. Cummings SR, Melton LJI (2002) Epidemiology and outcomes if osteoporotic fractures. Lancet 359(9319):1761-1767

4. Delmas PD, van de Langerijt L, Watts NB et al (2005) Underdiagnosis of vertebral fractures is a worldwide problem: the IMPACT study. J Bone Miner Res 20(4):557-563

5. Fink HA, Milavetz DL, Palermo L et al (2005) What proportion of incident radiographic vertebral deformities is clinically diagnosed and vice versa? J Bone Miner Res 20(7):1216-1222

6. Cooper C, Atkinson EJ, O'Fallon WM, Melton LJ III (1992) Incidence of clinically diagnosed vertebral fractures: a populationbased study in Rochester, Minnesota, 1985-1989. J Bone Miner Res 7(2):221-227

7. Gehlbach SH, Bigelow C, Heimisdottir M et al (2000) Recognition of vertebral fracture in a clinical setting. Osteoporos Int 11(7):577582

8. Gallagher JC, Genant HK, Crans GG, Vargas SJ, Krege JH (2005) Teriparatide reduces the fracture risk associated with increasing number and severity of osteoporotic fractures. J Clin Endocrinol Metab 90(3):1583-1587

9. Black DM, Arden NK, Palermo L et al (1999) Prevalent vertebral deformities predict hip fractures and new vertebral deformities but not wrist fractures. Study of Osteoporotic Fractures Research Group. J Bone Miner Res 14(5):821-828

10. Gallacher SJ, Gallagher AP, McQuillian C et al (2007) The prevalence of vertebral fracture amongst patients presenting with nonvertebral fractures. Osteoporos Int 18(2):185-192

11. Howat I, Carty D, Harrison J, Fraser M, McLellan AR (2007) Vertebral fracture assessment in patients presenting with incident nonvertebral fractures. Clin Endocrinol 67(6):923-930

12. Greenspan SL, von Stetten E, Emond SK, Jones L et al (2001) Instant vertebral assessment: a noninvasive dual X-ray absorptiometry technique to avoid misclassification and clinical mismanagement of osteoporosis. J Clin Densitom 4:373-380

13. van der Velde R, Takouhi O, Dumitrescu B et al (2015) Performance of statistical models of shape and appearance for semiautomatic segmentations of spinal vertebrae T4-L4 on digitized vertebral fracture assessment images. Spine J 15(6):12481254

14. Genant HK, Wu CY, van Kuijk C, Nevitt MC (1993) Vertebral fracture assessment using a semiquantitative technique. J Bone Miner Res 8(9):1137-1148

15. Center JR, Bliuc D, Nguyen TV, Eisman JA (2007) Risk of subsequent fracture after low-trauma in men and women. JAMA 297(4): 387-394

16. Waterloo S, Nguyen T, Ahmed LA et al (2012a) Important risk factors and attributable risk of vertebral fractures in the population-based Tromso study. BMC Musculoskelet Disord 13: 163

17. Seeman E, Bianchi G, Khosla S, Kanis J, Orwoll R (2006) Bone fragility in men — where are we? Osteoporos Int 17(11):1577-1583

18. Waterloo S, Nguyen T, Ahmed LA, Center JR, Morseth B, Nguyen ND, Eisman JA, Sogaard AJ, Emaus N (2012b) Important risk factors and attributable risk of vertebral fractures in the population-based Tromso study. BMC Musculoskelet Disord 13: 163

19. O'Neil TW, Felsenberg D, Varlow J, Cooper C, Kanis JA, Silman AJ (1996 Jul) The prevalence of vertebral deformity in European men and women: the European Vertebral Osteoporosis Study. J Bone Miner Res 11(7):1010-1018

20. Joes G (1996) Prevalent vertebral deformities in elderly men and women. Osteoporos Int 6(3):233-239

21. Jager PL, Jonkman S, Koolhaas W, Stiekema A et al (2011) Combined vertebral fracture assessment and bone mineral density measurement: a new standard in the diagnosis of osteoporosis in academic populations. Osteoporos Int 22:1059-1068

22. Schuit SC, van der Klift M, Weel AE, de Laet CE, Burger H, Seeman E, Hofman A, Uiitterlinden AG, van Leeuwen JP, Pols HA (2004) Fracture incidence and association with bone mineral density in elderly men and women: the Rotterdam Study. Bone 34: 195-202

23. Li N, Li X, Xu L, Sun, W, Cheng X and Tian W. Comparison of QTC and DXA: osteoporosis detection rates in postmenopausal women. Int J Endocrinol Vol 2013, Article ID 895474. doi:10. 1155/2013/895474

24. Netelenbos JC, Lems WF, Geussens PP et al (2009) Spine radiographs to improve the identification of women at high risk for fractures. Osteoporosis Int 20:1347-1352

25. Middleton ET, Steel SA (2008) Routine versus targeted vertebral fracture assessment for the detection of vertebral fractures. Osteoporos Int 19:1167-1173

26. Kuet K-P, Charlesworth D, Peel NFA (2013) Vertebral fracture assessment scans enhance targeting of investigations and treatment within a fracture risk assessment pathway. Osteoporos Int 24:10071014

27. Ferrar L, Jiang G, Schousboe J, DeBold CR, Eastell R (2008) Prevalent vertebral fracture: agreement between different readers, imaging modalities, and diagnostic approaches. J Bone Miner Res $23: 417-424$ 